\title{
Multi-axial fatigue life assessment of high speed car body based on PDMR method
}

\author{
Chaotao Liu ${ }^{1, *}$, Pingbo $\mathrm{Wu}^{1}$, and Fansong $\mathrm{Li}^{1}$ \\ ${ }^{1}$ Traction Power State Key Laboratory, Southwest Jiaotong University, No. 111, North 1st Section of Second Ring Road, \\ Jinniu District, Chengdu City, Sichuan Province, PRC
}

\begin{abstract}
This article mainly introduces a method of converting the acceleration signal of body bolster obtained by the circuit test into the load of the air spring seat of vehicle body. This method mainly decomposes the body's movement posture into the form of ups and downs, roll and nod. Then formulate the test plan according to the performance of the body fatigue test bench. The vertical and horizontal displacements and longitudinal force are used as control commands.Taking advantage of vehicle body fatigue test bench to reproduce these basic types of vibration. Establish the transfer function of the acceleration of the bolster and the displacement excitation of the air spring, and then obtaining the load of the air spring seat. Finally, the multi-axial fatigue life assessment of the vehicle body was performed using the obtained load combined with the Moment of Load Path Method and the Path-Dependent Maximum Range Method.
\end{abstract}

\section{Introduction}

Car body is an important bearing structure of high speed train. The key of car body anti-fatigue design is extracting the stress of measuring-point accurately. Simulation analysis and experimental verification is an essential part of the vehicle design stage. Simulation analysis has the advantages of high efficiency, low cost, etc. The test verification not only can verify the accuracy of the simulation calculation model, but also can take into account the incomplete technical considerations in the simulation analysis.

In this paper, firstly obtains the time history of acceleration at the car body bolster through the line test. The time domain signal of the acceleration is decomposed and compressed according to the operating posture of the vehicle body (sinking, rolling, nodding, etc.). Then under laboratory conditions, using the vehicle fatigue test bench to reproduce the actual vibration of the vehicle body. At the same time, the stress at the danger point and the load at loading point are continuously measured. Finally, the dynamic stress response of the vehicle body finite element model is calculated using the load data of loading point, and the fatigue life of the high-speed train body is predicted using PDMR and MLP methods.

\section{Determination of the test program}

In order to obtain the loading data of the loading point, first of all, the vibration of the vehicle body needs to be reproduced through the test. During the test, the high-speed EMU body is supported on two six-degreeof-freedom platforms. Each platform can realize the translation in three directions along $\mathrm{x}, \mathrm{y}$, and $\mathrm{z}$ and the rotation in three directions. The connection between the vehicle body and the six-degree-of-freedom platform is set according to the actual force status of the vehicle body. The position of the air spring seat only bears the vertical load, and the position of the center pin bears the lateral and longitudinal loads.

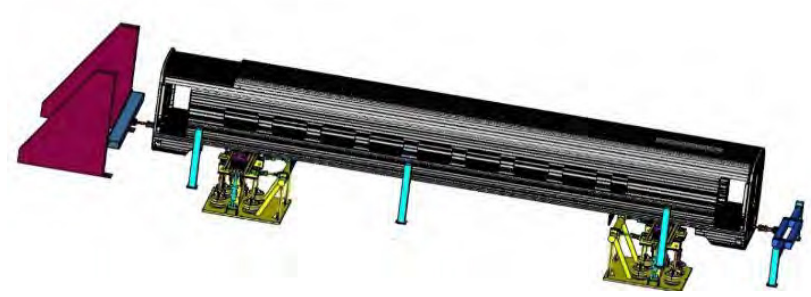

Fig. 1. Overall test program

Secondary vertical loading: Tooling for loading is installed on the six-degree-of-freedom platform. It releases lateral and longitudinal freedom through lateral and longitudinal linear bearings. The force sensor is arranged at the junction of the tooling and the test piece, and the rubber pad with smaller stiffness is arranged in the position of the vehicle-body interface, so as to avoid a rigid impact during the test.

\footnotetext{
Corresponding author: liuchaotao@live.cn
} 


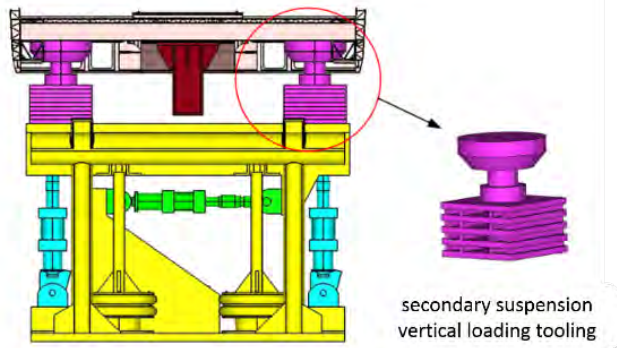

Fig. 2. Secondary suspension vertical loading tooling

The center pin loading tooling is also installed on the six-degree-of-freedom platform, and they are connected through a spherical hinge in order to release the vertical freedom.

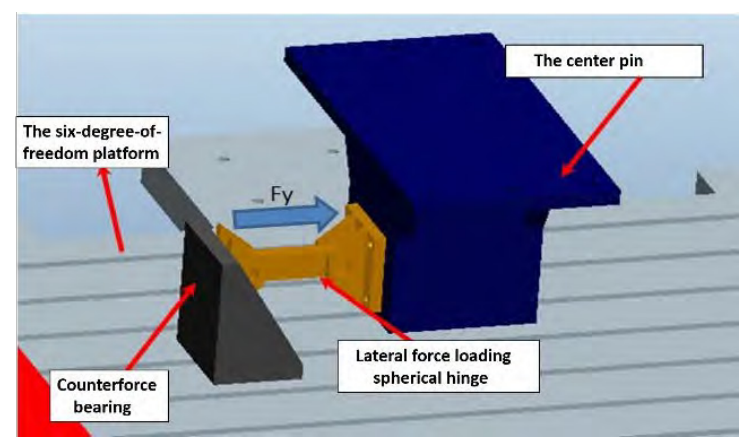

Fig. 3. The center pin loading tooling

Longitudinal actuators are arranged at the two coupler positions, which is used to simulate the coupler force.

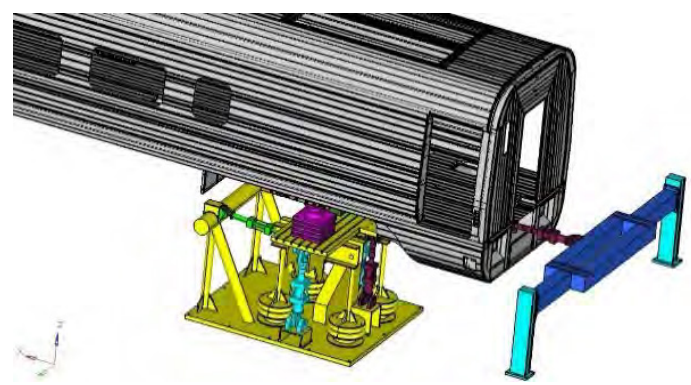

Fig. 4. Coupler force loading device

Each six-degree-of-freedom platform is equipped with two longitudinal actuators in the longitudinally and horizontal position for balancing the unbalanced longitudinal forces between the two couplers. The actuator of each platform is orthogonal decoupled in order to realize simultaneous loading of longitudinal, lateral and vertical position at different frequencies.

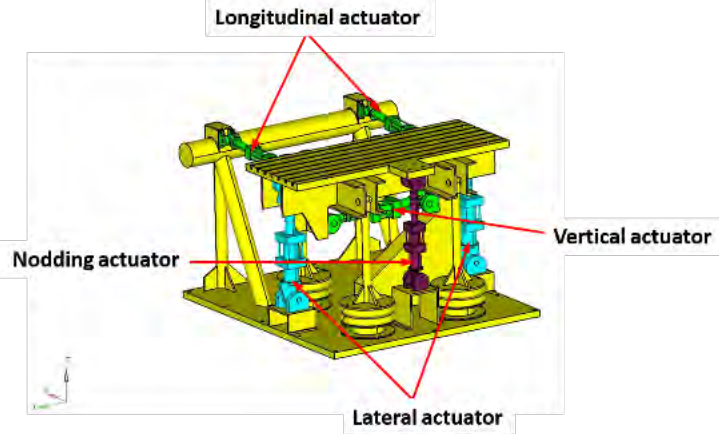

Fig. 5. Six-degree-of-freedom platforms

The above test load is loaded by force on longitudinal load, that is, the actuator is controlled by force, and the lateral and vertical loads are loaded by inertia, which are controlled by displacement.

\section{Determination of fatigue test load}

Reliable external load is the key of fatigue test. At present there are some international standards specifies the fatigue loads, such as EN 12663-2010 Railway application - Structural requirements of railway vehicle bodies ${ }^{[1]}$ (EN12663-2010 for short), JIS E 7106-2006 Rolling stock-General requirements of car body ${ }^{[2]}$ (JIS7106 for short), VDV152-2016 Recommendations on the Design for Strength of Urban Rail Rolling Stock according to BOStrab ${ }^{[3]}$ (VDV152-2016 for short) and so on. All of these standards stipulate the body's fatigue load through the vibration acceleration of the vehicle body, but these standards are mainly formulated for the operating environment of the relevant countries. So the fatigue loads are not suitable for the track conditions in China. Moreover, these loads are accelerated fatigue tests, and does not appear in the actual operating state under different working conditions of the vehicle body.

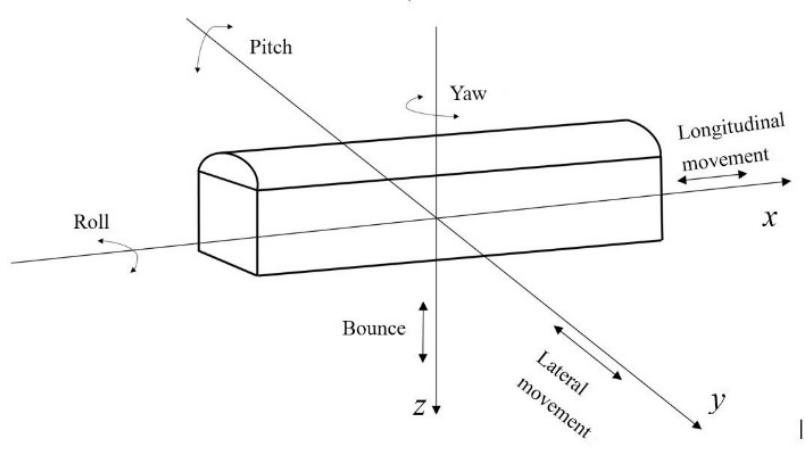

Fig. 6. The coordinate position of the body in space

Through the bench test, we can reproduce the operation posture of the vehicle body under different conditions, which is not only conducive to the research of the fatigue strength of the existing vehicle body, but also to the development of the new vehicle. A vehicle with spring suspension is a multi-degree-of-freedom vibration system. The spatial position of the vehicle 
body consists entirely of linear motion in three directions along $\mathrm{x}, \mathrm{y}, \mathrm{z}$ and gyration motion in three directions. Therefore, the movement of the vehicle body consists of floating movement, lateral movement, telescopic movement, yaw movement, nod movement and roll movement.

It is assumed that the body's acceleration of bouncing is $a_{1}(t)$, the acceleration of pitching is $a_{2}(t)$, the acceleration of rolling is $a_{3}(t)$, the lateral acceleration is $a_{4}(t)$, the acceleration of yawing is $a_{5}(t)$ and the longitudinal acceleration is $a_{6}(t)$. Four threeway accelerometers are arranged on both sides of the two bolsters, and four three-way accelerometers are arranged on the air spring seats of the two bogie frames. When the contribution of lateral roll to lateral acceleration is not considered, there is:

$$
\begin{gathered}
a_{1}(t)+a_{2}(t)-a_{3}(t)=a_{z 1}(t) \\
a_{1}(t)+a_{2}(t)+a_{3}(t)=a_{z 2}(t) \\
a_{1}(t)-a_{2}(t)-a_{3}(t)=a_{z 3}(t) \\
a_{4}(t)+a_{5}(t)=a_{y 1}(t) \\
a_{4}(t)-a_{5}(t)=a_{y 3}(t)
\end{gathered}
$$

Solved by:

$$
\begin{aligned}
& a_{1}(t)=\frac{a_{z 2}(t)+a_{z 3}(t)}{2} \\
& a_{2}(t)=\frac{a_{z 1}(t)-a_{z 3}(t)}{2} \\
& a_{3}(t)=\frac{a_{z 2}(t)-a_{z 1}(t)}{2} \\
& a_{4}(t)=\frac{a_{y 1}(t)+a_{y 3}(t)}{2} \\
& a_{5}(t)=\frac{a_{y 1}(t)-a_{y 3}(t)}{2}
\end{aligned}
$$

Where $a_{z 1}(t), a_{z 2}(t), a_{z 3}(t), a_{y 1}(t)$ and $a_{y 3}(t)$ are measured accelerations.

According to the test plan in section 1, the driving document for determining the vertical and lateral test loads is displacement excitation. The four vertical actuators of the two six-degree-of-freedom platform can simulate the three types of movements of the carbody's bouncing, rolling, and pitching, and two lateral actuators can simulate the yawing and rock-rolling of the body. Since the displacement of each actuator is relatively small when reproducing the running posture of the vehicle body, the coupling between the actuators is ignored.

Taking the vertical load as an example, in order to simulate the running conditions of a $350 \mathrm{~km} / \mathrm{h}$ straightline operation of a high-speed EMU, the acceleration of bouncing, rolling and pitching are calculated by taking 100 s test data according to the above formula. In the normal operation, due to the vehicle body is supported on the air spring, high-frequency loads cannot be transmitted to the vehicle body and the rigid body modes of the vehicle body are all within $2 \mathrm{~Hz}^{[4]}$, thus, the acceleration signal collected by the position of the bolster beam is low-pass filtered by $20 \mathrm{~Hz}$. The filtered data is subjected to FFT transformation to obtain the basic frequency $f_{1}$ of the body bouncing motion, the basic frequency $f_{2}$ of the rolling motion and the basic frequency $f_{3}$ of the pitching movement.

It is assumed that the acceleration of the body bounce is $1 \mathrm{~m} / \mathrm{s}^{2}$ when the vibration amplitudes of the four vertical actuators of the six-degree-of-freedom platform are $d_{1}$ and the frequency is $f_{1}$. When the two vertical actuators at the front end and two vertical actuators at the end apply an anti-phase displacement excitation $d_{2}$, and frequency is $f_{2}$, the pitching acceleration is $1 \mathrm{~m} / \mathrm{s}^{2}$. When the two vertical actuators at the left side end and two vertical actuators at the right side apply an anti-phase displacement excitation $d_{3}$, and frequency is $f_{3}$, the rolling acceleration is $1 \mathrm{~m} / \mathrm{s}^{2}$. The swaying and yawing are also processed in the same way.

It is assumed that the test system is linearly timedomain-invariant in order to get the fatigue test driver file. Based on the above transfer coefficient, the correspondence between the acceleration response of the body's bouncing, rolling and pitching movement and the positional displacement excitation of the air spring can be obtained. When simulating rolling of the vehicle body, the distance $l_{1}$ between the two vertical actuators is different from the distance $l_{2}$ between the air spring seats of the bogie, therefore, the displacement of the vertical actuator is $l_{1} d_{2} a_{2}(t) / l_{2}$ when the displacement of the air spring seat is excited by $d_{2} a_{2}(t)$.

Table 1. the relationship between motivation and response.

\begin{tabular}{|c|c|c|c|}
\hline $\begin{array}{c}\text { Body } \\
\text { movement }\end{array}$ & $\begin{array}{c}\text { Vibration } \\
\text { acceleration }\end{array}$ & $\begin{array}{c}\text { Displacement } \\
\text { excitation }\end{array}$ & $\begin{array}{c}\text { Vibration } \\
\text { frequency } \\
\text { Hz })\end{array}$ \\
\hline bounce & $a_{1}(t)$ & $d_{1} a_{1}(t)$ & $f_{1}$ \\
\hline Bottom & $a_{2}(t)$ & $l_{1} d_{2} a_{2}(t) / l_{2}$ & $f_{2}$ \\
\hline Left & $a_{3}(t)$ & $d_{3} a_{3}(t)$ & $f_{3}$ \\
\hline Right & $a_{1}(t)$ & $d_{1} a_{1}(t)$ & $f_{1}$ \\
\hline
\end{tabular}

Through the above table, the displacement excitation of the four vertical actuators can be obtained by:

$$
\begin{aligned}
& d_{z 1}(t)=d_{1} a_{1}(t)+l_{1} d_{2} a_{2}(t) / l_{2}-d_{3} a_{3}(t) \\
& d_{z 2}(t)=d_{1} a_{1}(t)+l_{1} d_{2} a_{2}(t) / l_{2}+d_{3} a_{3}(t) \\
& d_{z 3}(t)=d_{1} a_{1}(t)-l_{1} d_{2} a_{2}(t) / l_{2}-d_{3} a_{3}(t) \\
& d_{z 4}(t)=d_{1} a_{1}(t)-l_{1} d_{2} a_{2}(t) / l_{2}+d_{3} a_{3}(t)
\end{aligned}
$$

For the obtained displacement excitation time history, the data are compressed by deleting the data that contribute less to the damage. The process of 
compression must maintain the phase relationship between each load to ensure that the medium and high loads are not deleted. The compressed displacement excitation is used as the driver file for body fatigue test. The data obtained by the force sensor feedback of the four air spring seat position $F_{z 1}(t) F_{z 2}(t), F_{z 3}(t), F_{z 4}(t)$ can be used as the load spectrum to calculate the body damage.

Table 2. Carbody rigid modal test results

\begin{tabular}{|c|c|c|}
\hline No. & Modal Shape & $\begin{array}{c}\text { Modal frequency } \\
(\mathbf{H z})\end{array}$ \\
\hline 1 & Lower body rolling & 0.600 \\
\hline 2 & Carbody bouncing & 0.762 \\
\hline 3 & Carbody pitching & 1.053 \\
\hline 4 & Carbody yawing & 1.138 \\
\hline 5 & Upper body rolling & 1.561 \\
\hline
\end{tabular}

\section{Calculation of vehicle body fatigue life}

The fatigue life of the vehicle body is evaluated by the load spectrum obtained in Section III. Since the main frequency of the load spectrum is much lower than the lowest frequency of the elastic modal of the vehicle body, the quasi-static method is used to calculate the fatigue life of the vehicle body. Under the effect of the loads of air springs, center pins, couplers, etc., the body structure is subjected to multi-axial cyclic stress during operation, and in particular, the position of the weld seam is often in a multi-axial stress state due to geometrical abrupt changes. Therefore, it is necessary to evaluate the multi-axial fatigue strength of key welds of high-speed train body structure.

The load on the car body is neither in-phase or proportional, and the fatigue damage caused by this nonproportional load is inextricably linked to the load path and material. In order to define the parameters and period of non-proportional variable amplitude multiaxial fatigue damage, Professor Dong has invented a loop counting method based on path-dependency which is called as PDMR (Path-Dependent Maximum Range). Two important parameters can be obtained by PDMR for the stress time courses of a vehicle body measuring point: the maximum effective stress range counted in each half cycle and the resulting path length. The vehicle body fatigue damage can be calculated by combining the two parameters generated by PDMR and a new kind of MLP(Moment of Load Path) also raised by Professor Dong.

\section{Test verification}

In order to verify the effectiveness of the above method, taking the body of a high-speed EMU as the research object, in order to restore the vibration of the same type of car body running on the WuhanGuangzhou line, the vehicle body vibration acceleration data on this line is used as a target for verification. The verification process mainly includes: building body fatigue test device according to test plan, using line test data to compile driver files according to the method presented here, fatigue test, extracting the load spectrum at the point of action and the dynamic stress at key measurement points. based on the load spectrum and using the PDMR method and MLP method for fatigue assessment of key measurement points. Finally, the measured damage values were compared with the simulation values to verify the effectiveness of the method.

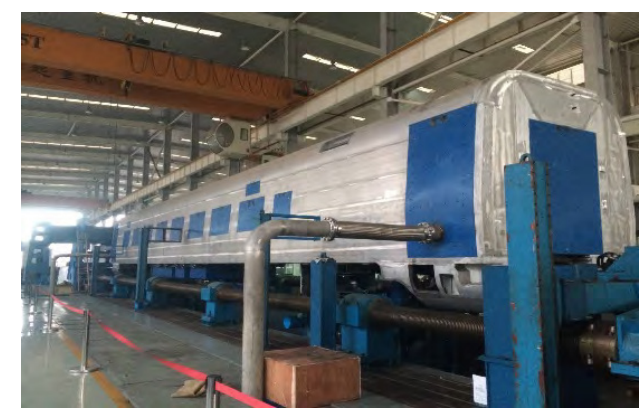

Fig. 7. Test devices

Due to the time, only the compilation of test devices and drive files has been completed, and the evaluation of the fatigue life of the vehicle body has not yet been carried out at present.

\section{Conclusion and outlook}

This article mainly proposes a method of converting the acceleration signal of the bolster beam position into the air spring position force signal. And put forward making use of MLP and PDMR method for the multiaxial fatigue life assessment of the car body. Due to the time, although this method has not been well verified, after the test is completed, the dynamic model of the test bench can be used to restore the test, and the load of the loading point under different working conditions can be restored through the virtual test. And then to achieve the evaluation of the car body fatigue life. Combined with flaw detection results, it is also possible to evaluate the remaining life of existing models.

\section{References}

1. EN 12663-2010 Railway application - Structural requirements of railway vehicle bodies

2. JIS E 7106-2006 Rolling stock-General requirements of car body 
3. VDV152-2016 Recommendations on the Design for Strength of Urban Rail Rolling Stock according to BOStrab

4. Fansong Li. Research on Vibration and Fatigue of Carbody for EMU under Service Environment. D., 60, (2018)

5. Dong P, Wei Z, Hong J K. A path-dependent cycle counting method for variable-amplitude multi-axial loading. J. International Journal of Fatigue, 720-734, (2010) 